\title{
The Matryoshka technique in percutaneous nephrolithotomy
}

\author{
Stefano Paolo Zanetti ${ }^{1}$, Matteo Fontana ${ }^{1}$, Elena Lievore ${ }^{1}$, Matteo Turetti ${ }^{1}$, Fabrizio Longo ${ }^{1}$, \\ Elisa De Lorenzis ${ }^{1,2}$, Giancarlo Albo ${ }^{1,2}$, Emanuele Montanari ${ }^{1,2}$ \\ ${ }^{1}$ Fondazione IRCCS Ca' Granda Ospedale Maggiore Policlinico, Department of Urology, Milan, Italy; \\ ${ }^{2}$ Department of Clinical Sciences and Community Health, University of Milan, Department of Urology, Milan, Italy.
}

\section{Summary Objective: Miniaturized percutaneous nephrolithotomy (PCNL) reduces the risk of} haemorrhagic complications, but the limited field of work represents a drawback. To obtain the best outcomes, the percutaneous access size should be intraoperatively tailored. Our purpose is to describe the indications and the procedural steps of the Matryoshka technique and to report its clinical outcomes. Materials and methods: We performed a retrospective analysis of the data from consecutive Matryoshka PCNL procedures from October 2016 to January 2018. Collected data included patients' history, stone characteristics, intra- and post-operative items, stone clearance and need for retreatment. The main indication to the Matryoshka technique is the inability to securely position a guidewire due to an obstruction or narrowness in the pyelocalyceal system. This technique begins by puncturing the calyx hosting the stone and advancing a hydrophilic guidewire through the needle. If the guidewire cannot proceed beyond the stone, the Matryoshka technique is employed for tract stabilization. The tract is carefully dilated with small-bore instruments and a cautious lithotripsy is performed to create enough space to introduce the guidewire beyond the stone under visual control. Once the access has been stabilized the surgeon can upsize the tract to the optimum to complete the procedure. Additionally, the technique can be employed when an intraoperative reassessment induces the surgeon to further dilate the tract to quicken the procedure. Results: Sixteen patients were included, with a median stone volume of $3.49 \mathrm{~cm}^{3}$. Median operative time was 112 minutes. Three Clavien I-II (postoperative fever) and one Clavien IIIB (colon perforation) complications were reported. No blood transfusions were recorded. Three patients underwent scheduled retreatment as part of a multistep procedure. Out of the remaining 13 patients, 10 (76.9\%) obtained a complete stone clearance. Conclusions: The Matryoshka technique helps the urologist to obtain a secure percutaneous access and makes PCNL flexible and progressive, potentially minimizing the risk of access-related complications.

KEY WORDS: Kidney stones; Percutaneous nephrolithotomy; Minimally invasive surgical procedures; Percutaneous nephrostomy.

Submitted 9 December 2020; Accepted 16 January 2021

\section{INTRODUCTION}

Percutaneous nephrolithotomy (PCNL) is the gold standard for the treatment of kidney stones larger than $20 \mathrm{~mm}$ (1), providing satisfactory stone-free rates (SFR) even in case of remarkable stone burden. Despite its effectiveness, the main drawbacks of this technique are its invasiveness and the significant risk of haemorrhagic complications, requiring a blood transfusion in around 7\% (range $0-20 \%$ ) of the cases (2). The site of the percutaneous renal puncture and the technique employed for the dilation of the access route are the most crucial steps to perform an effective and safe procedure (3-4). In the last decades, the introduction of small calibre instruments contributed to reduce the complications rate (5), making PCNL safer. However, miniaturized techniques added a few limitations, including reduced visibility, scarce instrument choice for lithotripsy and lapaxy, longer operative times and elevated renal pelvic pressures, restricting the applicability of these techniques to smallto-medium sized stones (6). In order to obtain the best outcomes, the percutaneous access size should be tailored to the distinctive characteristics of both the stone and the patient. This principle allows the urologist to minimize the morbidity of the treatment, enhancing at the same time its effectiveness. The minimally invasive PCNL (MIP) set, devised by Nagele (7), includes various progressive size nephroscopes and access sheaths in a single system. This modular set can be employed to intraoperatively adjust the tract size to the characteristics of the stones and to the anatomy of the collecting system, and to overcome potentially dangerous accessrelated issues. This allows to combine the advantages of miniaturized and standard PCNL: we defined this concept as the "Matryoshka technique" (8).

The aim of this study is to describe the indications and the procedural steps of the "Matryoshka technique" in PCNL and to report the clinical outcomes from our experience with this approach.

\section{Materials and Methods}

Study design

The PCNL database of our tertiary referral stone centre was retrospectively reviewed, and all consecutive patients who underwent a Matryoshka PCNL from October 2016 to January 2018 were included in this study. All procedures were carried out by a single expert urologist (more than 1000 PCNLs performed) in the 
same centre. Collected data included patients' anthropometrics and medical and surgical history, stone characteristics, intra- and post-operative items, complications, stone clearance and need for retreatment.

Comorbidities were graded according to the Charlson Comorbidity Index. All patients underwent a preoperative contrast-enhanced CT scan to evaluate the stone characteristics (laterality, stone number and location, total volume and mean density expressed in Hounsfield Units) and to plan the surgical approach. The stone volume was measured by means of the ellipsoid formula ( $\mathrm{a} \times \mathrm{b} \times \mathrm{c} \times \pi / 6)$ and in case of multiple stones, total stone volume was calculated as the sum of the volumes of the single stones. Intraoperative data included the number of percutaneous tracts employed, the successful placement of a safety guidewire, the access sheath size, lithotripsy modality, intraoperative complications, exit strategy and operative time, defined as the time from the kidney puncture to the exit strategy. The Matryoshka technique was evaluated both in terms of indication and tract size upscaling. Postoperative items included haemoglobin drop, need for blood transfusions, estimated glomerular filtration rate (eGFR) change and length of hospital stay. Postoperative complications were graded according to the PCNL-adjusted Clavien Score (9). Stone clearance was assessed through follow-up imaging (CT scan or ultrasound) performed 3-6 months after surgery and was defined as the total absence of residual fragments.

All methods were carried out in accordance with relevant guidelines and regulations. Informed consent was obtained from all patients or from the legal guardians for patients having age less than 18 . This study was approved by the local Ethics Committee (Comitato Etico Milano Area 2). Data were collected and analysed using the statistical software SPSS 25.0 (IBM Cor., Armonk, NY, USA).

\section{Indications}

The main indication to the Matryoshka technique in PCNL is the inability to safely pass a guidewire into the renal collecting system without risking disrupting the calyx, due to the presence of a stone occupying the punctured calyx (calyceal staghorn) or its infundibulum (stone-engaged infundibulum), possibly causing the calyx to retain urine (stone-engaged hydrocalyx). In each one of these cases, the lack of a securely positioned guidewire could render the access ineffective and could cause several access-related complications. To minimize these risks, a less disruptive, smaller size tract should be initially employed until the setting is carefully evaluated and a safety guidewire is positioned under visual control, so that the access can be stabilized and upsized.

The indication to the Matryoshka technique may be posed preoperatively if the urographic phase of the CT scan shows a narrow calyceal infundibulum hosting the stone or a calyceal staghorn stone, but more often it becomes clear during the procedure, when, after puncturing the target calyx, the guidewire cannot proceed beyond the stone in the renal pelvis and down the ureter. Furthermore, the Matryoshka technique can be employed in those situations in which the guidewire has properly passed, but the surgeon prefers to assess the local anatomy and the actual stone load before dilating the tract.

\section{Armamentarium}

The armamentarium to perform a Matryoshka PCNL doesn't differ from that of a classic PCNL. What is essential, though, is the availability of two or more different size nephroscopes and access sheaths to perform a progressive access. At least the first scope and sheath should be miniaturized, in order to start the procedure in the least invasive possible way. The availability of different size instruments allows the surgeon to shift from a smaller to a larger access when the local conditions demand and allow it. In our series we used the MIP set (Karl Storz SE, Tuttlingen, Germany). The complete MIP set is composed of three nephroscopes (7.5 Ch., $12 \mathrm{Ch}$., 19.5 Ch.), and by a series of compatible dilators and metallic percutaneous sheaths, categorized in the extra-small (XS, 9,5 Ch.), small (S, 12 Ch.), medium (M, 16-22 Ch.) and large (L, 24-26 Ch.) subsets (8).

\section{Technique}

As the traditional PCNL, the Matryoshka procedure starts by placing a ureteral catheter up to the renal pelvis and by performing a retrograde pyelogram. If the preoperative urographic CT scan or the pyelogram show a staghorn or an infundibular stone, possibly obstructing the calyceal neck, the setting for the Matryoshka technique is defined.

Subsequently, after placing the patient in the supine Valdivia position, the calyx hosting the stone is punctured under fluoroscopic and/or ultrasonographic control and an hydrophilic guidewire is advanced through the needle. If the calyceal neck is narrow or obstructed and the guidewire cannot proceed beyond the stone (Figure 1A), the Matryoshka technique can be employed for tract stabilization. The tract is carefully dilated with small-bore dilators and sheaths (MIP XS, S or M subsets), and a compatible nephroscope is introduced. Once the stone has

\section{Figure 1.}

Representation of the Matryoshka technique.

(A) Stone obstructing the calyceal neck, preventing the guidewire from being inserted;

(B) Cautious lithotripsy through a small access until creating enough space for the guidewire(s) to pass;

(C) Tract dilation to the needed or allowed size according to the anatomical relation between the calyx and the stone; (D) Further tract dilation in order to speed up the procedure in case of very large stones with favourable anatomy.

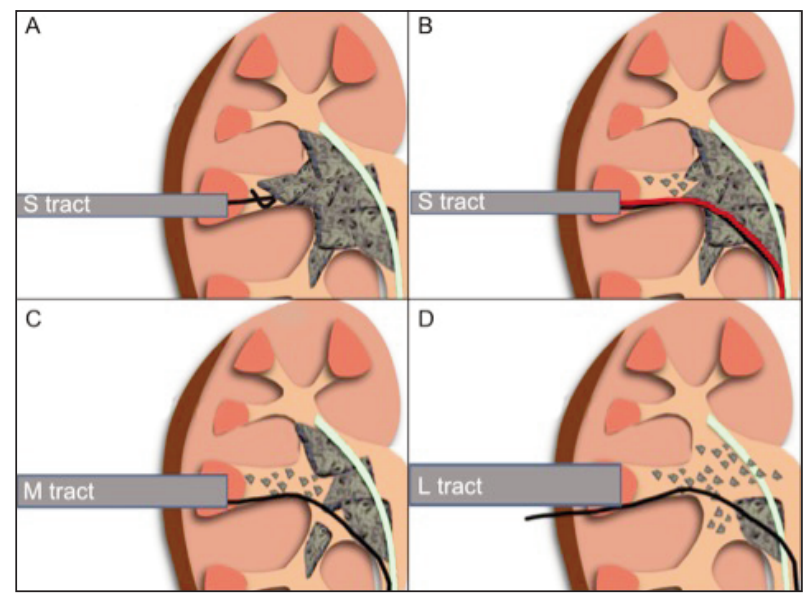


been visualized, cautious Holmium:YAG laser lithotripsy is started, creating enough space for the guidewire to pass, under visual control, beyond the stone into the renal pelvis and possibly down the ureter (Figure 1B). Since it is always possible to accidentally displace or kink the working guidewire during further manoeuvres and progressive tract dilations, the use of a safety guidewire is recommended as it minimizes the risk of losing the tract. When the access has been stabilized and the anatomical relation between the calyx and the stone is assessed, the surgeon can choose the dilation size needed or allowed, taking care not to dilate the tract beside the stone and not to force the calyx to a diameter exceeding its anatomy (Figure 1C). This is the key concept of the Matryoshka technique. This technique can also be employed in case of a narrow or partially obstructed calyceal neck irrespective of the secure placement of a working guidewire, or when a miniaturized access is initially chosen to limit PCNL morbidity but nephroscopic evaluation after partial lithotripsy reveals enough space to further dilate the tract. In this situation, it is possible to upsize the tract to the largest calibre that is respectful of the pyelocalyceal anatomy to speed up the procedure. After the secondary dilation is performed, lithotripsy can be continued. An upsized tract offers a wider choice of lithotripsy probes, better irrigation, clearer vision and lower intrapyelic pressures. In case of very large stones, the tract diameter can be further upscaled according to intraoperative needs (Figure 1D). After lithotripsy, fragments are evacuated through the vacuum cleaner effect, a nitinol basket or endoscopic graspers. At the end of the procedure, a nephrostomy tube is placed, when needed, over the safety guidewire.

\section{RESULTS}

The Matryoshka technique was applied in 16 out of 74 procedures $(21.6 \%)$ performed at our institution in the study period. Patients' and stones' characteristics are described in Table 1. In the $75 \%$ of the cases, multiple stones were treated. Median total stone volume was 3.49 $\mathrm{cm}^{3}$ (IQR 1.81-5.02). Intraoperative items and postoperative outcomes are reported in Table 2. Median operative time was 112 minutes (IQR 91-130). The indication to the Matryoshka technique was access stabilization in 14 cases (87.5\%), due to the inability to advance the guidewire beyond the stone because of a calyceal staghorn stone (six cases), a stone-engaged calyceal infundibulum (four cases) or a stone-engaged hydrocalyx (four cases). In the remaining two cases (12.5\%), intraoperative reassessment induced the surgeon to upsize the access to speed up and complete the procedure. In all the cases, through the Matryoshka technique, we managed to position a safety guidewire and obtain a secure access to the collecting system. Three patients reported postoperative fever (Clavien I-II) and one experienced a colonic puncture that was managed by temporary loop colostomy (Clavien IIIB). Median hemoglobin drop was -1.6 g/dL (IQR -1.3 - -2.2), and none of the patients experienced a hemorrhagic complication or received a blood transfusion. We registered no complications regarding urinary leakage or drainage. Concerning stone clearance, the overall stone free rate was $62.5 \%$ (10 out of 16 patients). Four patients needed a
Table 1.

Patients' and stones' characteristics.

\begin{tabular}{|c|c|}
\hline $\begin{array}{ll}\text { Sex n }(\%) & \\
& \text { Male } \\
& \text { Female }\end{array}$ & $\begin{array}{l}11(68.7 \%) \\
5(31.3 \%)\end{array}$ \\
\hline Age (years) mean $( \pm$ SD) & $51.4(13.7)$ \\
\hline BMI $\left(\mathrm{kg} / \mathrm{m}^{2}\right)$ mean $( \pm \mathrm{SD})$ & $26.0(3.4)$ \\
\hline \multicolumn{2}{|l|}{ Charlson Comorbidity Index n (\%) } \\
\hline $\begin{array}{l}0-1 \\
\geq 2\end{array}$ & $\begin{array}{l}12(75 \%) \\
4(25 \%)\end{array}$ \\
\hline \multicolumn{2}{|l|}{ History of ipsilateral stone treatment $\mathbf{n}(\%)$} \\
\hline $\begin{array}{l}\text { SWL } \\
\text { URS } \\
\text { PCNL } \\
\text { Pyelolithotomy } \\
\text { Any treatment } \\
\text { None }\end{array}$ & $\begin{array}{c}4(25 \%) \\
2(12.5 \%) \\
3(18.8 \%) \\
2(12.5 \%) \\
6(37.5 \%) \\
10(62.5 \%)\end{array}$ \\
\hline \multicolumn{2}{|l|}{ Laterality $\mathbf{n}(\%)$} \\
\hline $\begin{array}{l}\text { Right } \\
\text { Left }\end{array}$ & $\begin{array}{l}8(50 \%) \\
8(50 \%)\end{array}$ \\
\hline \multicolumn{2}{|l|}{ Stone number $\mathrm{n}(\%)$} \\
\hline $\begin{array}{l}\text { Single } \\
\text { Multiple }\end{array}$ & $\begin{array}{l}4(25 \%) \\
12(75 \%)\end{array}$ \\
\hline Stone volume $\left(\mathrm{cm}^{3}\right)$ median (IQR) & $3.49(1.81-5.02)$ \\
\hline Stone mean HU value (HU) median (IOR) & $907(834-1003)$ \\
\hline
\end{tabular}

\section{Table 2.}

Intraoperative items and postoperative outcomes.

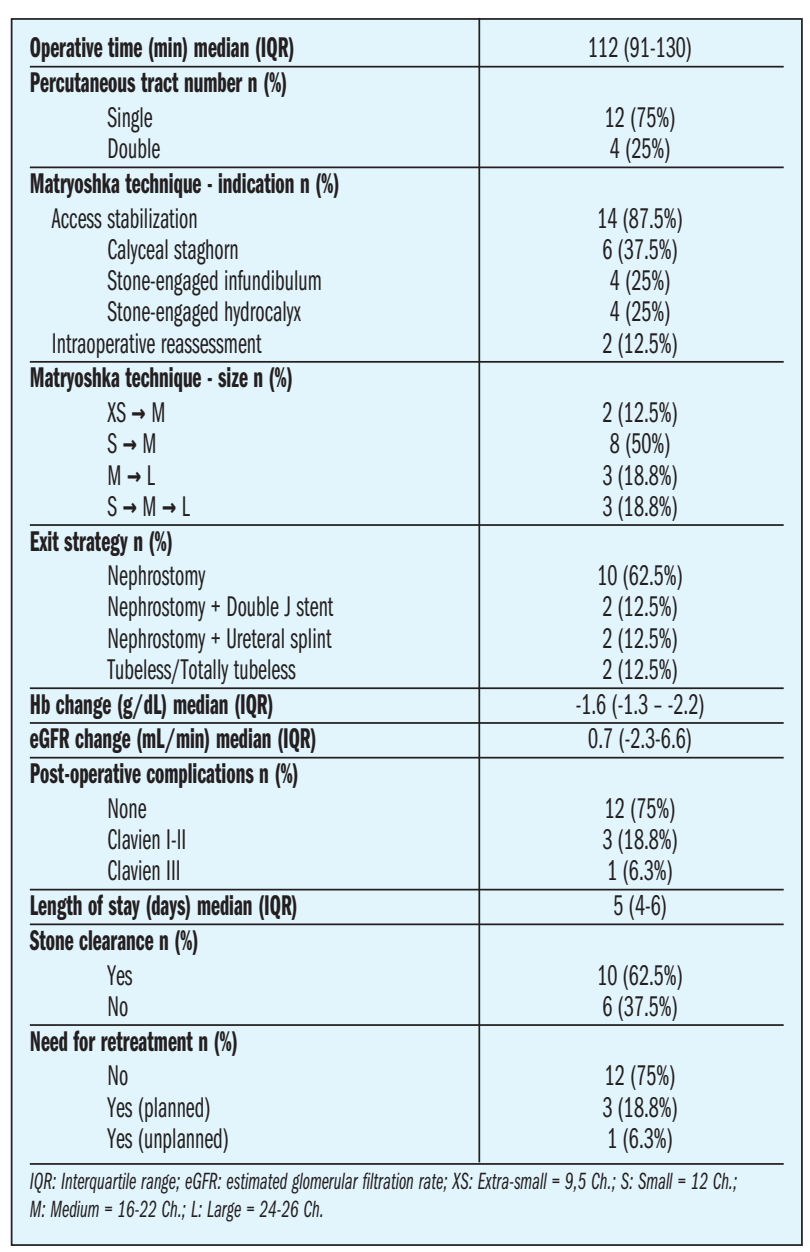


Table 3.

Matryoshka procedures.

\begin{tabular}{|c|c|c|c|c|}
\hline Patient & Stone load & Percutaneous tract & Matryoshka Indication & Matryoshka size \\
\hline N.S.,,+35 yrs. & 18.9 cm³ $^{3}$ HU max 1278; HU mean 681 staghorn (pelvis, middle calyx, lower calyx) & 2 tracts (lower calyx) & Calyceal staghorn & 17.5 Ch. $\rightarrow 22$ Ch. \\
\hline R.P., o', 45 yrs. & 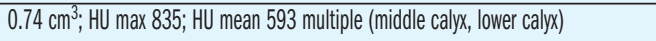 & 2 tracts (lower calyx) & Stone-engaged infundibulum & 12 Ch. $\rightarrow 16$ Ch. \\
\hline U.L., $0^{\pi}, 38$ yrs. & $3.80 \mathrm{~cm}^{3}$; HU max 592; HU mean 458 multiple (pelvis, lower calyx) & 2 tracts (lower calyx) & Stone-engaged infundibulum & 9.5 Ch. $\rightarrow 17.5$ Ch. \\
\hline O.P.,,, 54 yrs. & $3.18 \mathrm{~cm}^{3}$; HU max 1415; HU mean 775 single (upper calyx) & 1 tract (upper calyx) & Stone-engaged hydrocalyx & $12 \mathrm{Ch} . \rightarrow 16 \mathrm{Ch}$. \\
\hline C.C., $0^{\pi}, 54$ yrs. & $1.44 \mathrm{~cm}^{3}$; HU max 1659; HU mean 997 multiple (middle calyx, lower calyx) & 1 tract (lower calyx) & Stone-engaged hydrocalyx & 12 Ch. $\rightarrow 16$ Ch. \\
\hline S.P., o,, 52 yrs. & $0.82 \mathrm{~cm}^{3}$; HU max 1646; HU mean 962 multiple (middle calyx) & 1 tract (middle calyx) & Stone-engaged hydrocalyx & $12 \mathrm{Ch} . \rightarrow 16 \mathrm{Ch}$. \\
\hline I.A., $९, 72$ yrs. & $3.53 \mathrm{~cm}^{3}$; HU max 1145; HU mean 900 staghorn (pelvis, lower calyx) & 1 tract (lower calyx) & Intraoperative reassessment & 12 Ch. $\rightarrow 16$ Ch. \\
\hline T.l.,, , 62 yrs. & $3.45 \mathrm{~cm}^{3}$; HU max 1256; HU mean 906 staghorn (pelvis, lower calyx) & 1 tract (lower calyx) & Intraoperative reassessment & 12 Ch. $\rightarrow 16$ Ch. \\
\hline S.T., $0^{7}, 56$ yrs. & $6.83 \mathrm{~cm}^{3}$; HU max 1045 ; HU mean 950 staghorn (pelvis, lower calyx) & 1 tract (lower calyx) & Stone-engaged infundibulum & 17.5 Ch. $\rightarrow 24$ Ch. \\
\hline B.S., ơ, 56 yrs. & $2.99 \mathrm{~cm}^{3}$; HU max 1380; HU mean 1150 staghorn (pelvis, lower calyx) & 2 tracts (lower and middle calyx) & Calyceal staghorn & $12 \mathrm{Ch} . \rightarrow 16 \mathrm{Ch}$. \\
\hline P.M., 0, 14 yrs. & 10.42 cm³; HU max 1049; HU mean 853 staghorn (pelvis, middle calyx, lower calyx) & 1 tract (lower calyx) & Calyceal staghorn & $12 \mathrm{Ch} . \rightarrow 17.5 \mathrm{Ch} . \rightarrow 24 \mathrm{Ch}$. \\
\hline C.C., $0^{7}, 53$ yrs. & $4.42 \mathrm{~cm}^{3}$; HU max; HU mean staghorn (pelvis, middle calyx, lower calyx) & 1 tract (middle calyx) & Calyceal staghorn & 12 Ch. $\rightarrow 17.5$ Ch. $\rightarrow 24$ Ch. \\
\hline C.S., $0^{n}, 65$ yrs. & $24.02 \mathrm{~cm}^{3}$; HU max 1619; HU mean 1200 staghorn (complete) & 1 tract (lower calyx) & Calyceal staghorn & 16 Ch. $\rightarrow 24$ Ch. \\
\hline M.E., $0^{x}, 49$ yrs. & $3.61 \mathrm{~cm}^{3}$; HU max 1285; HU mean 908 staghorn (pelvis, lower calyx) & 1 tract (lower calyx) & Calyceal staghorn & 12 Ch. $\rightarrow 17.5$ Ch. $\rightarrow 24$ Ch.. \\
\hline
\end{tabular}

retreatment, which was already scheduled as part of a planned multistep procedure for three of them. Ten out of the 13 patients scheduled for a single step procedure (76.9\%) obtained a complete stone clearance.

The single procedures are presented in Table 3, with details regarding the stone load, the Matryoshka technique indication and the access tracts size.

\section{Discussion}

The authors of a recent review on miniaturized PCNL stated that the surgical treatment that each patient should be offered ought to be tailored to the distinctive characteristics of both the patient and the stone (10). The Matryoshka technique is the extremization of this concept: the opportunity to intraoperatively individualize the tract diameter to the anatomy of the pyelocalyceal system enables the surgeon to take advantage of the benefits of the different sized scopes and sheaths in every single procedural step. To the best of our knowledge, this is the first report of a series of patients treated with the Matryoshka technique. The main benefit of this technique is to provide a more secure percutaneous approach to the pyelocalyceal system in case of several access-related issues that could potentially undermine both the safety and proficiency of the tract creation.

In order to safely dilate the access and to position a percutaneous sheath, a guidewire should be firmly set inside the urinary tract: this manoeuvre is crucial to minimize the risk of kinking or slipping of the guidewire itself, that would render the access difficult or ineffective, and to reduce the mobility of the punctured kidney. When the guidewire cannot be securely held in the pyelocalyceal system, it is prudent to establish the smallest possible tract, which can be created with less strain on the guidewire and the kidney, and it is less harmful than a larger one. After the initial nephroscopy and the subsequent individuation of the issue underlying the suboptimal placement of the guidewire, the route for its advancement can be created by means of a prudent lithotripsy. Once the guidewires are firmly positioned, the tract can be dilated to the desired calibre.

In addition, the initial assessment of the urinary tract through a miniaturized nephroscope allows a precise recognition of anatomical variations, enabling the surgeon to preserve the integrity of the pyelocalyceal system: for instance, the identification of a narrow calyceal infundibulum could compel the urologist to complete the PCNL with small bore instruments, possibly preventing inadvertent disruptions of the urinary tract. Flexibility and scalability are two more strengths of this technique, which allows the reassessment of the local conditions during the procedure and the modification of the surgical approach in case of need. With this concept in mind, the chosen instruments can be readjusted on the basis of the characteristics of both the stone and the patient, and a truly tailored approach can be achieved.

The results from our initial experience seem to support these concepts. Although we treated complex cases, with significant stone loads and a high prevalence of multiple, staghorn stones, none of the patients experienced an haemorrhagic complication or required a blood transfusion; moreover, no cases of postoperative urine extravasation were recorded. Additionally, with the employment of the Matryoshka technique, all of the gained accesses have been successfully stabilized and exploited, and none was rendered ineffective because of guidewire slipping or kinking. Although we registered a colonic perforation, we do not believe that this complication is related to the Matryoshka technique in itself, as it generally occurs during the primary tract creation and not during secondary dilations, that are performed on an already established tract. Specifically, the perforation we reported occurred during the initial access despite ultrasonographic control, in a paraplegic patient that suffered from severe bowel disfunction and enlargement. Regarding our stone free rate, we believe it can be deemed satisfactory considering that the Matryoshka technique is 
especially useful in complex and challenging cases, usually characterized by less favourable results.

Our work is not devoid of limitations. First of all, our sample is quite meagre: this is due to the particular indications of the Matryoshka technique, that was applied in a limited number of patients in the considered period of time. Secondly, even if the results are encouraging, our work lacks a control group, that we believe would be hard to identify because of the unusual characteristics of the cases treated. Additionally, randomizing patients would be extremely difficult, as the indication to our technique is frequently defined intraoperatively.

\section{Conclusions}

The Matryoshka technique appears to be a safe and effective approach to obtain a secure percutaneous access to the kidney in challenging situations. It makes the PCNL procedure flexible and progressive, allowing the surgeon to intraoperatively adjust the tract size according to the local anatomy and stone characteristics. In this way, the less invasive achievable tract is stabilized, tailored to the patient and the stone, potentially minimizing the chances of calyceal tear and the related risk of complications.

\section{REFERENCES}

1. Türk C, Skolarikos A, Neisius A, et al. EAU Guidelines on Urolithiasis. 2019
2. Seitz C, Desai M, Häcker A, et al. Incidence, Prevention, and Management of Complications Following Percutaneous Nephrolitholapaxy. Eur Urol. 2012; 61:146-158.

3. Tahra A, Sobay R, Bindayi A, et al. Papillary vs non-papillary access during percutaneous nephrolithotomy: Retrospective, matchpaired case-control study. Arch Ital Urol Androl. 2020; 92:50-52.

4. Sahin A, Uruc F. The comparative analysis of the three dilatation techniques in percutaneous nephrolithotomy: Which one is safer? Arch Ital Urol Androl. 2019; 91:171-173.

5. Ruhayel Y, Tepeler A, Dabestani S, et al. Tract sizes in miniaturized percutaneous nephrolithotomy: a systematic review from the European Association of Urology Urolithiasis Guidelines Panel. Eur Urol. 2017; 72:220-235.

6. Heinze A, Gozen AS, Rassweiler J. Tract sizes in percutaneous nephrolithotomy: does miniaturization improve outcome? Curr Opin Urol. 2019; 29:118-123.

7. Nagele U, Schilling D, Anastasiadis AG, et al. Minimally invasive percutaneous nephrolitholapaxy (MIP). Urol - Ausgabe A. 2008; 47:1066-1073

8. Zanetti SP, Boeri L, Gallioli A, et al. Minimally invasive PCNL MIP. Arch Esp Urol. 2017; 70:226-234.

9. De La Rosette JJMCH, Opondo D, Daels FPJ, et al. Categorisation of complications and validation of the Clavien score for percutaneous nephrolithotomy. Eur Urol. 2012; 62:246-255.

10. Proietti S, Giusti G, Desai M, Ganpule AP. A Critical Review of Miniaturised Percutaneous Nephrolithotomy: Is Smaller Better? Eur Urol Focus. 2017; 3:56-61.

\section{Correspondence}

Stefano Paolo Zanetti, MD (Corresponding Author)

stefano.p.zanetti@gmail.com

Matteo Fontana, MD

teo.fontana@yahoo.it

Elena Lievore, MD

elena.lievore01@gmail.com

Matteo Turetti, MD

matteo.turetti@gmail.com

Fabrizio Longo, MD

longomd@gmail.com

Elisa De Lorenzis, MD

elisa.delorenzis@gmail.com

Giancarlo Albo, MD

albo.giancarlo@gmail.com

Emanuele Montanari, MD

emanuele.montanari@unimi.it

Via Della Commenda 15, 20122, Milano, Italy 